\title{
An annotated checklist of the Syncarida (Crustacea, Malacostraca) of the world
}

\author{
ANA I. CAMACHO \\ Museo Nacional de Ciencias Naturales, CSIC, Dept. Biodiversidad y Biología Evolutiva, C/ José Gutiérrez \\ Abascal 2, 28006-Madrid, Spain.
}

\section{Table of contents}

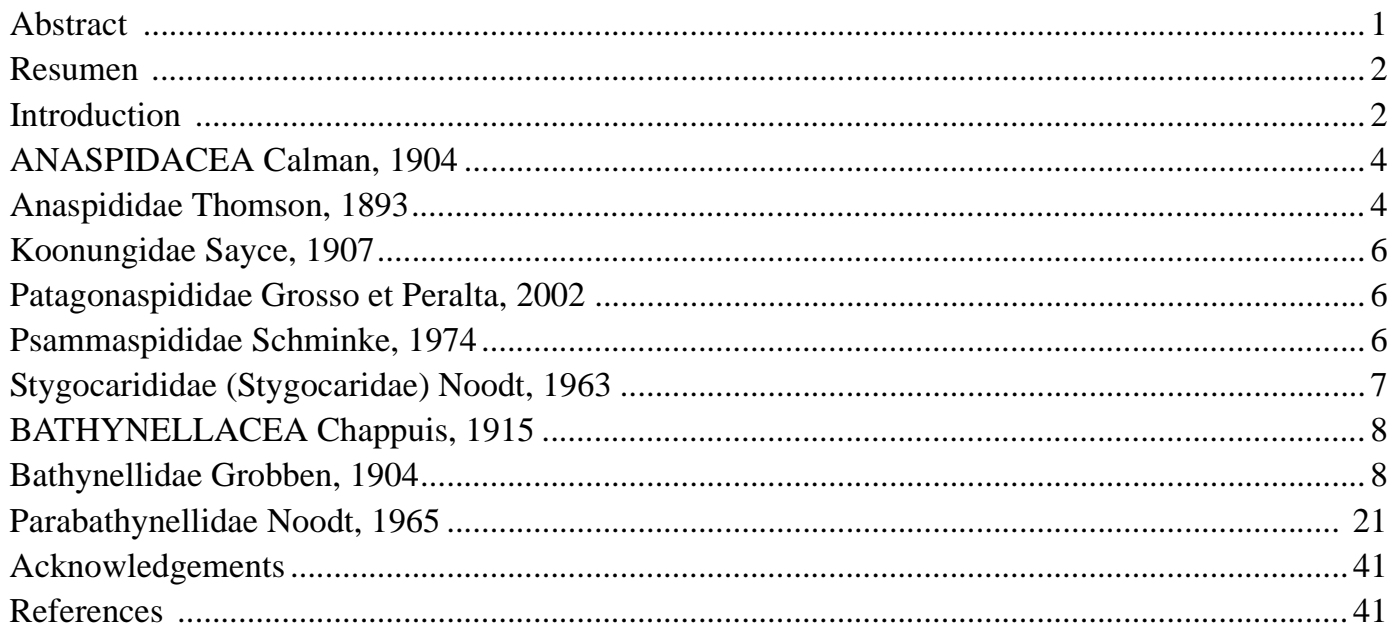

\begin{abstract}
An annotated list of the 256 species and subspecies of Syncarida known to occur in the world is presented, including synonymies, information on habitat type (caves, springs, wells, etc.), type locality, other localities where the taxa have been found, and an abbreviated reference to the original description and other important taxonomic references when available. Critical remarks about the validity of some taxa are included. A summary of genera and species known per continent and a map of the world distribution of genera is presented. The work includes a comprehensive list of syncarid literature.
\end{abstract}

Key words: Crustacea, Syncarida, species list, distribution 